\title{
First-principles prediction of oxygen octahedral rotations in perovskite-structure $\mathrm{EuTiO}_{3}$
}

\author{
Konstantin Z. Rushchanskii, ${ }^{1}$ Nicola A. Spaldin, ${ }^{2}$ and Marjana Ležaić ${ }^{1}$ \\ ${ }^{1}$ Peter Grünberg Institut, Quanten-Theorie der Materialien, Forschungszentrum Jülich and JARA, 52425 Jülich, Germany \\ ${ }^{2}$ Materials Theory, Department of Materials, ETH Zurich, Wolfgang-Pauli-Strasse 27, CH-8093 Zurich, Switzerland \\ (Received 6 October 2011; revised manuscript received 20 December 2011; published 26 March 2012)
}

\begin{abstract}
We present a systematic first-principles study of the structural and vibrational properties of perovskite-structure $\mathrm{EuTiO}_{3}$. Our calculated phonon spectrum of the high-symmetry cubic structural prototype shows strong M- and R-point instabilities, indicating a tendency to symmetry-lowering structural deformations composed of rotations and tilts of the oxygen octahedra. Subsequent explicit study of 14 different octahedral tilt-patterns showed that the $I 4 / \mathrm{mcm}$, Imma, and $R \overline{3} \mathrm{c}$ structures, all with antiferrodistortive rotations of the octahedra, have significantly lower total energy than the prototype $P m \overline{3} m$ structure. We discuss the dynamical stability of these structures, and the influence of the antiferrodistortive structural distortions on the vibrational, optical, and magnetic properties of $\mathrm{EuTiO}_{3}$, in the context of recent unexplained experimental observations.
\end{abstract}

DOI: 10.1103/PhysRevB.85.104109

PACS number(s): 77.84.-s, 71.15.Mb, 78.30.-j

\section{INTRODUCTION}

Europium titanate, $\mathrm{EuTiO}_{3}$, was the first ternary compound of divalent $\mathrm{Eu}^{2+}$ to be identified. ${ }^{1,2}$ It is a G-type antiferromagnet (AFM) with the low magnetic ordering temperature of $5.3 \mathrm{~K}$, consistent with the highly localized $4 f$ electrons on the $\mathrm{Eu}^{2+}$ ions. ${ }^{1,3,4}$ Until recently, its structural ground state was thought to be the ideal cubic perovskite structure, with $\operatorname{Pm} \overline{3} m$ symmetry. ${ }^{2} \mathrm{EuTiO}_{3}$ is insulating with a high dielectric constant $(\sim 400)$ at low temperature, indicating quantum paraelectric behavior and proximity to ferroelectric instability. ${ }^{5}$ Strong interactions between the magnetic and dielectric properties have been reported ${ }^{5-9}$ including evidence for an unusual third-order coupling. ${ }^{10}$ It has been suggested that $\mathrm{EuTiO}_{3}$ is the prototype for studying quantum paraelectric behavior in magnetic systems. ${ }^{11}$

In addition to these experiments on bulk $\mathrm{EuTiO}_{3}$, there has been a series of recent studies on $\mathrm{EuTiO}_{3}$ thin films ${ }^{12-15}$ motivated in part by the subsequently verified prediction that epitaxial strain should induce ferroelectricity and multiferroicity. ${ }^{16,17}$ Likewise "chemical strain," i.e., doping at the A site with larger divalent ions, has been shown to induce ferroelectricity, e.g., in alloys with $\mathrm{BaTiO}_{3} .{ }^{18}$ The latter is particularly intriguing, as multiferroic $(\mathrm{Eu}, \mathrm{Ba}) \mathrm{TiO}_{3}$ ceramics fulfill the requirements for a solid state search for the electric dipole moment of electron. ${ }^{19}$

In this work, we revisit bulk $\mathrm{EuTiO}_{3}$, with a particular focus on the possibility that the ground state is not, in fact, the high-symmetry $\operatorname{Pm} \overline{3} m$ perovskite prototype, but a lower symmetry structure possessing rotations or tilts of the oxygen octahedra. Until recently, all computational studies of $\mathrm{EuTiO}_{3}$ had neglected this possibility. ${ }^{16,20,21}$ We mentioned recently in Ref. 19 that in the course of calculating the properties of $(\mathrm{Eu}, \mathrm{Ba}) \mathrm{TiO}_{3}$, we noticed a calculated antiferrodistorted ground state for $\mathrm{EuTiO}_{3}$. Then, in Ref. 17, the influence of such possible oxygen octahedra tiltings on the induced polarization in strained $\mathrm{EuTiO}_{3}$ was discussed. Finally, a recent study ${ }^{22}$ confirmed experimentally the presence of a structural phase transition in $\mathrm{EuTiO}_{3}$ using specific heat measurements, and suggested, in analogy to $\mathrm{SrTiO}_{3}$, that the lowtemperature phase should have oxygen rotations and $I 4 / \mathrm{mcm}$ symmetry.
Our present ab initio study of $\mathrm{EuTiO}_{3}$ was motivated by a series of experimental papers ${ }^{23,24}$ in which infrared (IR) reflectivity and time-domain terahertz transmission spectra of $\mathrm{EuTiO}_{3}$ ceramics yielded intriguing results that can be better understood if the crystalline symmetry is lower then $P m \overline{3} m$. The studies $^{23,24}$ found three polar optical phonons between 6-600 $\mathrm{K}$, typical for the perovskite structure. Analysis of the oscillator strengths (or equivalently, the mode-plasma frequencies) and comparison of their values with those of known perovskite prototypes such as $\mathrm{PbTiO}_{3}$ (with ferroelectrically active Aand $\mathrm{B}$-site cations) and $\mathrm{BaTiO}_{3}$ (with active B-site cations) suggested that (i) the lowest-energy transverse-optical (TO) phonon TO1 corresponds predominantly to the Slater mode, ${ }^{25}$ with opposite vibration of the Ti cations and the oxygen octahedra (see Fig. 1, left); softening of this mode dominates the quantum paraelectric behavior, (ii) the second lowest-energy TO2 phonon consists of vibrations of the Eu cation against the $\mathrm{TiO}_{6}$ octahedra, commonly known as the Last mode, (see Fig. 1, center), and (iii) the highest frequency TO4 (the TO3 mode is not IR active) phonon represents oxygen octahedral bending, usually called an Axe mode (see Fig. 1, right).

However, some features observed in the experiment are not understood: (i) a significant temperature dependence of the oscillator strength was found for the TO1 and TO2 modes, indicating changes in the vibrational mode eigenvectors with temperature. The changes are most apparent in the temperature range 250-400 K (see Fig. 5 in Ref. 24), with the oscillator strength for the TO1 mode decreasing and for the TO2 mode increasing with increasing temperature. (ii) The width of the peak in the imaginary dielectric function $\epsilon^{\prime \prime}$ that is associated with the soft TO1 mode increases when temperature is lowered. This is unusual, because at low-temperature, anharmonic effects that could give rise to the widening of the peak in $\epsilon^{\prime \prime}$ usually decrease [see Fig. 3(b) in Ref. 24]. (iii) The mode at around $420 \mathrm{~cm}^{-1}$ in the IR reflectivity spectrum is temperature dependent (see Fig. 2 in Ref. 24). However, the $\mathrm{Eu}_{2} \mathrm{Ti}_{2} \mathrm{O}_{7}$ pyrochlore phase, to which this peak was tentatively assigned, does not show a structural phase transition.

In this work, we present a detailed density-functional theory based study of the structural and vibrational properties 


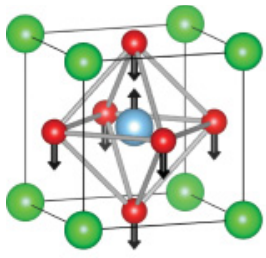

(a)

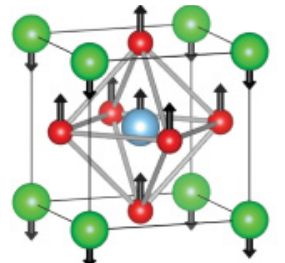

(b)

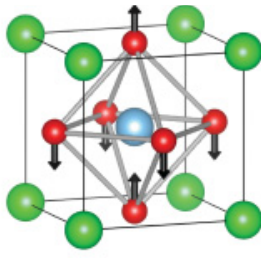

(c)
FIG. 1. (Color online) Schematic diagrams ${ }^{25}$ of the atomic displacements in the (a) slater mode in which the B-site cation moves in the opposite direction from the rigid oxygen octahedral cage, (b) Last mode in which the A-site cation moves opposite to the rigid $\mathrm{BO}_{6}$ cage, and (c) Axe mode in which the apical oxygens move opposite to the in-plane oxygens and the A- and B-site cations do not participate; a deformation of the oxygen cage results.

of antiferromagnetic $\mathrm{EuTiO}_{3}$. Our main finding is that the ground state is not in fact the high-symmetry $P m \overline{3} m$ structure (in analogy with other perovskite materials, for example $\mathrm{KNbO}_{3}{ }^{26}$ ), but a lower-symmetry phase with tilts and rotations of the oxygen octahedra. The calculated properties of the lower-symmetry phase are more consistent with the measured behavior than those calculated for the high-symmetry $P m \overline{3} m$ structure. In addition, we obtain a strong magnetophonon interaction in our new ground-state structure. The remainder of this paper is organized as follows: in Sec. II, we describe the technical details of our calculations, Sec. III contains the results of our calculations of the structural and dynamical properties of $\mathrm{EuTiO}_{3}$ and a new interpretation of the IR optical properties along with the influence of magnetic fields. We conclude in Sec. IV.

\section{CALCULATION DETAILS}

We carried out first-principles density-functional calculations within the spin-polarized generalized gradient approximation (GGA). ${ }^{27}$ For the electronic structure calculations and structural relaxations, we used projector augmented-wave potentials as implemented in Vienna $a b$ initio simulation package (VASP). ${ }^{28-31}$ We considered the following valenceelectron configuration: $5 s^{2} 5 p^{6} 4 f^{7} 6 s^{2}$ for $\mathrm{Eu}, 3 s^{2} 3 p^{6} 3 d^{2} 4 s^{2}$ for $\mathrm{Ti}$, and $2 s^{2} 2 p^{4}$ for oxygen.

To account for the strong electron correlation effects on the $f$ shells of Eu atoms, we used the DFT $+U$ scheme $^{32}$ in Dudarev's approach ${ }^{33}$ with an on-site Coulomb parameter $U=5.7 \mathrm{eV}$ and Hund's exchange $J_{H}=1.0 \mathrm{eV}$ after Ref. 17. We have checked our main conclusions (i.e., the stability of the antiferrodistorted phases relative to the cubic phase) for several values of these parameters $(U=4$ to $8 \mathrm{eV})$ as well as without the $\mathrm{LDA}+U$ correction and obtained the same result. We used a kinetic energy cutoff of $500 \mathrm{eV}$ and a $6 \times 6 \times 6(4 \times 4 \times 4) \Gamma$-centered $k$-point mesh for the unit cell (supercell) simulations. Spin-orbit interaction was not taken into account. The experimental lattice parameter of the cubic phase of $\mathrm{EuTiO}_{3}, 3.90 \AA$, ${ }^{1,3,5}$ was used instead of the theoretical value of $3.943 \AA$. For structural relaxations that involved changes of the unit cell shape, we performed a fixed-volume relaxation to suppress the known GGA overestimation of the lattice volume. In the structural relaxations, we minimized Hellman-Feynman forces to 0.5
$\mathrm{meV} / \AA$. To investigate the structural stability, we utilized $2 \times 2 \times 2$ supercells containing 40 atoms. This allowed us to investigate all possible structural distortions that can originate from the zone-boundary instabilities in a systematic way and to minimize the error in total energy calculations when comparing energies of the different structures. For each symmetry investigated, the initial ideal perovskite structure was distorted according to the corresponding oxygen octahedral rotation pattern, and then the internal atomic positions and lattice parameters were relaxed until the convergence criteria were reached.

The calculations of the dynamical properties were performed using the force-constant method. ${ }^{34,35}$ To investigate possible dynamical instabilities we used a $2 \times 2 \times 2$ supercell of the primitive five-atom $\mathrm{EuTiO}_{3}$ unit cell; this supercell size allows most common oxygen octahedra tilting and rotational patterns as well as the likely commensurate AFM magnetic orders. The Hellman-Feynman forces were calculated for displacements of atoms of up to $0.04 \AA$. The dynamical matrix for each $q$ point in the Brillouin zone was constructed by Fourier transforming the force constants calculated at the $\Gamma$ point and the Brillouin zone boundaries. Phonon-mode frequencies and atomic displacement patterns for each $q$ point were obtained as eigenvalues and eigenvectors of the dynamical matrices. Born effective charges for symmetryinequivalent ions in the simulation cell ${ }^{36,37}$ were calculated with the Berry-phase technique. ${ }^{38}$

\section{RESULTS AND DISCUSSION}

\section{A. Properties of the high-symmetry $P m \overline{\mathbf{3}} m$ structure}

In the following, we show that assuming $P m \overline{3} m$ is the ground-state structure leads to discrepancies with existing experiments in the nature of the long-wavelength phonon modes and in the mode-plasma frequencies. Because of the notorious uncertainty in DFT lattice constants, we investigate whether these discrepancies can be removed by varying the unit cell volume. We find that a partial agreement (in the mode-plasma frequencies) can be reached, but only if the lattice parameter is increased to unphysical values at which the lattice is unstable with respect to the polar displacements. Furthermore, our analysis of the full phonon spectrum of $P m \overline{3} m$ structure indicates strong antiferrodistortive instablities.

First, we calculated the zone-center phonon wave numbers and eigenvectors in cubic $\mathrm{EuTiO}_{3}$ (see Table I) and obtained results consistent with other recent calculations. ${ }^{16}$ As we mentioned above, in Ref. 24, it was suggested that the TO1

TABLE I. Slater, Last, and Axe modes decomposition (in \%) of the soft polar mode TO1 and second-energy polar mode TO2 for two lattice parameters of cubic $\mathrm{EuTiO}_{3}$ perovskites.

\begin{tabular}{lcccc}
\hline \hline Mode & $\omega, \mathrm{cm}^{-1}$ & Slater & Last & Axe \\
\hline Cell parameter $a=3.90 \AA:$ & & & \\
TO1 & 60 & 60 & 37 & 3 \\
TO2 & 153 & 61 & 38 & 1 \\
Cell parameter $a=3.95 \AA:$ & & & \\
TO1 & $96 i$ & 88 & 12 & \\
TO2 & 123 & 27 & 72 & 1 \\
\hline \hline
\end{tabular}


TABLE IIa. Experimental and theoretical values of the phonon wave numbers and mode-plasma frequencies (both quantities in $\mathrm{cm}^{-1}$ ) for the crystalline structures of $\mathrm{EuTiO}_{3}$ with $P m \overline{3} \mathrm{~m}, I 4 / \mathrm{mcm}$, and $R \overline{3} c$ space groups, with ferromagnetic (FM) and G-type antiferromagnetic (AFM) magnetic ordering on the A-site cations. Mode-plasma frequency values are given in italics. TO1, TO2, and TO4 label the optically active modes in the ideal perovsite structure; these modes are split in the tilted structures.

\begin{tabular}{|c|c|c|c|c|c|c|c|c|c|c|c|c|c|c|c|c|c|}
\hline \multirow{3}{*}{$\frac{\text { No. }}{1}$} & \multirow{3}{*}{$\begin{array}{c}\text { Mode } \\
\text { TO1 }\end{array}$} & \multicolumn{4}{|c|}{ Exp. $^{a}$} & \multicolumn{4}{|c|}{$P m \overline{3} m$} & \multicolumn{4}{|c|}{$I 4 / \mathrm{mcm}$} & \multicolumn{4}{|c|}{$R \overline{3} c$} \\
\hline & & \multicolumn{2}{|c|}{$10 \mathrm{~K}$} & \multicolumn{2}{|c|}{$400 \mathrm{~K}$} & \multicolumn{2}{|c|}{ AFM } & \multicolumn{2}{|c|}{ FM } & \multicolumn{2}{|c|}{ AFM } & \multicolumn{2}{|c|}{ FM } & \multicolumn{2}{|c|}{ AFM } & \multicolumn{2}{|c|}{$\mathrm{FM}$} \\
\hline & & 82 & 1550 & 125 & 1250 & 67 & 1289 & 60 & 1327 & 107 & 1323 & 101 & 1394 & 80 & 1300 & 72 & 1353 \\
\hline 2 & & & & & & & & & & 128 & 1607 & 118 & 1622 & 124 & 1500 & 117 & 1540 \\
\hline 3 & TO2 & 153 & 400 & 159 & 860 & 155 & 925 & 153 & 864 & 154 & 898 & 153 & 813 & 153 & 565 & 152 & 452 \\
\hline 4 & & & & & & & & & & 156 & 314 & 156 & 239 & 160 & 984 & 157 & 911 \\
\hline 5 & & & & & & & & & & 164 & & 218 & & 164 & & 147 & \\
\hline 6 & & & & & & & & & & 251 & 40 & 251 & 45 & 242 & 200 & 241 & 188 \\
\hline 7 & & & & & & & & & & 419 & 250 & 419 & 242 & 419 & 233 & 419 & 241 \\
\hline 8 & TO4 & 539 & 619 & 540 & 625 & 537 & 824 & 536 & 820 & 523 & 732 & 522 & 729 & 514 & 737 & 513 & 732 \\
\hline 9 & & & & & & & & & & 531 & 718 & 530 & 715 & 533 & 733 & 532 & 728 \\
\hline 10 & & & & & & & & & & 802 & & 805 & & 803 & & 804 & \\
\hline
\end{tabular}

${ }^{\mathrm{a}}$ Experimental data from Ref. 24.

mode is mainly Slater type, based on the observation that the measured value of the mode-plasma frequency for this mode is in the same range as for typical ferroelectric perovskites with active B-site sublattices such as $\mathrm{BaTiO}_{3} .{ }^{25}$ In contrast to the

TABLE IIb. Phonon wave numbers and mode-plasma frequencies (both quantities in $\mathrm{cm}^{-1}$ ) for the orthorhombic Imma phase of $\mathrm{EuTiO}_{3}$ with ferromagnetic (FM) and antiferromagnetic (AFM) ordering on the A-site cations. Mode-plasma frequency values are given in italics. The meaning of the TO1, TO2, and TO4 labels is the same as in Table IIa.

\begin{tabular}{|c|c|c|c|c|c|}
\hline \multirow{2}{*}{$\frac{\text { No. }}{1}$} & \multirow[t]{2}{*}{ Mode } & \multicolumn{2}{|c|}{ AFM } & \multicolumn{2}{|c|}{ FM } \\
\hline & & 36 & & 34 & \\
\hline 2 & & 40 & & 49 & \\
\hline 3 & TO1 & 98 & 1318 & 85 & 1414 \\
\hline 4 & & 104 & & 104 & \\
\hline 5 & & 106 & & 105 & 1479 \\
\hline 6 & & 110 & & 107 & \\
\hline 7 & & 110 & 1233 & 112 & \\
\hline 8 & & 131 & 1596 & 119 & 1609 \\
\hline 9 & TO2 & 154 & 822 & 152 & 656 \\
\hline 10 & & 155 & 201 & 155 & 816 \\
\hline 11 & & 159 & 945 & 156 & 107 \\
\hline 12 & & 164 & & 166 & \\
\hline 13 & & 237 & 134 & 235 & 134 \\
\hline 14 & & 249 & & 247 & \\
\hline 15 & & 283 & 122 & 282 & 76 \\
\hline 16 & & 413 & & 412 & \\
\hline 17 & & 414 & & 415 & \\
\hline 18 & & 416 & 224 & 418 & 167 \\
\hline 19 & & 418 & 230 & 422 & 302 \\
\hline 20 & & 424 & & 424 & \\
\hline 21 & & 436 & & 434 & \\
\hline 22 & & 491 & & 486 & \\
\hline 23 & & 499 & & 498 & \\
\hline 24 & TO4 & 516 & 730 & 513 & 716 \\
\hline 25 & & 523 & 736 & 524 & 743 \\
\hline 26 & & 537 & 725 & 535 & 707 \\
\hline 27 & & 801 & & 802 & \\
\hline
\end{tabular}

suggestion made in Ref. 24, however, our analysis of the mode eigenvectors shows that the low-energy TO1 mode has a rather high contribution from Eu displacements, rather than being a straightforward Slater mode. In Table I, we decompose our calculated eigendisplacements of atoms in the TO1 and TO2 modes (calculated at the experimental lattice constant) into the Slater, Last, and Axe modes discussed in Ref. 24. In addition to providing a comparison with the earlier interpretation, such a decomposition provides information on the relative activities of the A- and B-site cations, and correspondingly the role of the two cationic sublattices, in the ferroelectric transition. Our analysis shows that both the TO1 and TO2 eigenvectors have, in fact, similar contributions from the Slater and Last modes. For the TO1 mode, we find that the Slater- and Last-like displacements are in phase, whereas they are out of phase for the TO2 mode. This leads to a significant difference in the observed as well as calculated (see below) values of the mode-plasma frequencies for both modes.

Next, to compare with the experimental information obtained from IR-reflectance spectra, ${ }^{24}$ we calculated the modeplasma frequencies for the TO1, TO2, and TO4 modes, using the following standard definition: ${ }^{25}$

$$
\left(\Omega_{m}\right)_{\alpha \alpha}=\frac{1}{\sqrt{\epsilon_{0} V_{0}}}\left|\sum_{k \beta}\left(Z_{k}^{*}\right)_{\alpha \beta} m_{k}^{-\frac{1}{2}}\left(x_{m}\right)_{k \beta}\right| .
$$

Here, $\left(\Omega_{m}\right)_{\alpha \alpha}$ is the mode-plasma frequency of mode $m$, polarized along direction $\alpha, \epsilon_{0}$ is the permittivity of vacuum, $V_{0}$ is the unit cell volume, $\left(Z_{k}^{*}\right)_{\alpha \beta}$ is the Born effective charge tensor of ion $k, m_{k}$ is the atomic mass of ion $k$, and $\left(x_{m}\right)_{k \beta}$ is the eigenvector of mode $m$. Eigenvectors are normalized to unity:

$$
\sum_{k \beta}\left(x_{m}\right)_{k \beta}^{2}=1
$$

We used the Born effective charges of 2.63|e| for $\mathrm{Eu}, 7.46|e|$ for $\mathrm{Ti},-2.18|e|$ for $\mathrm{O}_{\perp}$, and $-5.74|e|$ for $\mathrm{O}_{\|}$, which we calculated at the experimental lattice constant of $3.90 \AA$ in our calculation of the mode-plasma frequencies. 
Interestingly, our calculated mode-plasma frequencies (see Table IIa) match well with the experimental values in the hightemperature (300-600 K) range, but are markedly different from the low-temperature experimental values (see Fig. 5 of Ref. 24). Since our DFT calculations do not include thermal effects, particularly the strong hardening of the soft mode found with increasing temperature in $\mathrm{EuTiO}_{3}$, we expect them instead to match the low-temperature values. As we saw earlier, the soft mode eigenvectors show significant volume dependence. Therefore to check whether the discrepancy lies in our choice of unit cell volume, we investigated the volume dependence of the key quantities entering Eq. (1). We found that the Born effective charges are largely insensitive to the volume: for values of the lattice constant $a$ between 3.8 and $4.0 \AA$, the Born effective charges change from 2.68 to $2.61|e|$ for $\mathrm{Eu}$, from 7.46 to $7.49|e|$ for $\mathrm{Ti}$, from -2.26 to $-2.13|e|$ for $\mathrm{O}_{\perp}$, and from -5.69 to $-5.83|e|$ for $\mathrm{O}_{\|}$. Therefore the changes in mode-plasma frequencies must be caused by the changes in the mode eigenvectors.

Next, we varied the lattice parameter to see if we could obtain good agreement between the calculated and lowtemperature experimental values of the mode-plasma frequencies. In fact, we found good agreement only by increasing $a$ to $3.95 \AA$. From Table I, we can see that at $a=3.95 \AA$, the TO1 mode is predominantly of Slater type, while the TO2 mode has Last character. ${ }^{39}$ However, the Slater mode becomes imaginary, indicating a polar structural instability not observed experimentally in $\mathrm{EuTiO}_{3}$ under normal conditions. Moreover, increasing the lattice parameter to $3.95 \AA$ is clearly not a physically reasonable choice: the lattice constant at low temperature (our DFT results correspond to $0 \mathrm{~K}$ ) should be smaller than that at room or higher temperature because of thermal expansion of the lattice.

Therefore we conclude that the observed discrepancy between the measured low-temperature mode-plasma frequencies and the calculated values is not due to an inappropriate choice of the lattice parameter in our calculations. Next, we explore further the low-temperature structural properties of $\mathrm{EuTiO}_{3}$ in attempt to explain the discrepancy.

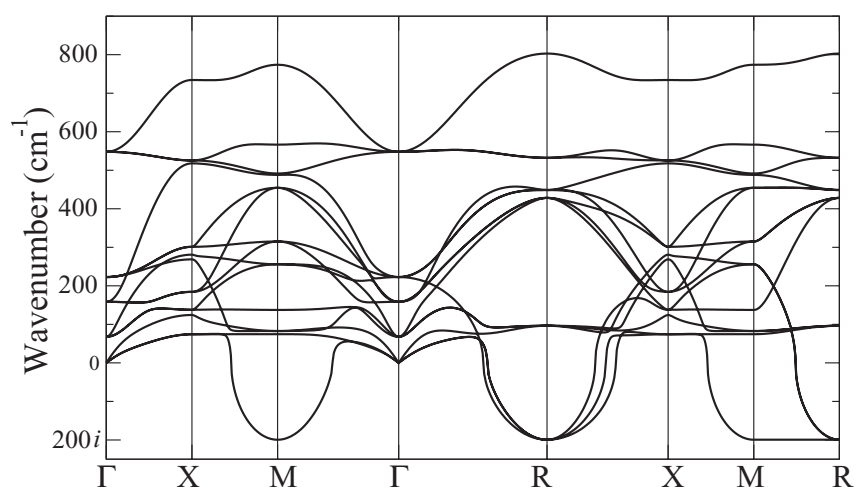

FIG. 2. Phonon spectrum of ideal cubic perovskite $\mathrm{EuTiO}_{3}$, calculated at the experimental volume with lattice parameter $a=$ $3.90 \AA$. The imaginary wave numbers of the low-lying modes at the Brillouin zone boundary $\mathrm{M}$ and $\mathrm{R}$ points indicate structural instabilities. Ferromagnetic ordering of Eu magnetic moments was used for computational convenience.
Our calculated phonon spectrum of $\mathrm{EuTiO}_{3}$ in the $P m \overline{3} m$ phase (see Fig. 2) indeed reveals strong structural instabilities at the $\mathrm{R}\left(\frac{1}{2}, \frac{1}{2}, \frac{1}{2}\right)$ and $\mathrm{M}\left(\frac{1}{2}, \frac{1}{2}, 0\right)$ high-symmetry points and along the M-R symmetry line. These instabilities are seen as modes with imaginary wave numbers and are similar to those found in calculations for $\mathrm{Pm} \overline{3} m \mathrm{SrTiO}_{3},{ }^{40}$ which is known experimentally to have the $I 4 / \mathrm{mcm}$ ground state with alternating rotations of the oxygen octahedra around the $c$ axis (Glazer notation ${ }^{41} a^{0} a^{0} c^{-}$). As in the case of $\mathrm{SrTiO}_{3}$, our calculated eigenvectors indicate that these instabilities are nonpolar and arise from the tilting and rotation of the oxygen octahedra. The eigenvector for the M-point instability shows in-phase rotations of the oxygen octahedra around one or more pseudocubic axes, whereas at the $\mathrm{R}$ point, octahedra rotate with alternating out-of-phase sense.

\section{B. Search for the structural ground state}

Motivated by our finding of rotational instabilities in ideal cubic perovskite $\mathrm{EuTiO}_{3}$, we next search for the structural ground state by comparing the calculated total energies of structures containing different combinations of rotations of the $\mathrm{TiO}_{6}$ octahedra. As categorized by Howard and Stokes, ${ }^{42}$ condensation of combinations of M- and R-point octahedral rotations in the perovskite structure can yield any of 15 possible symmetry space groups (including ideal perovskite structure),

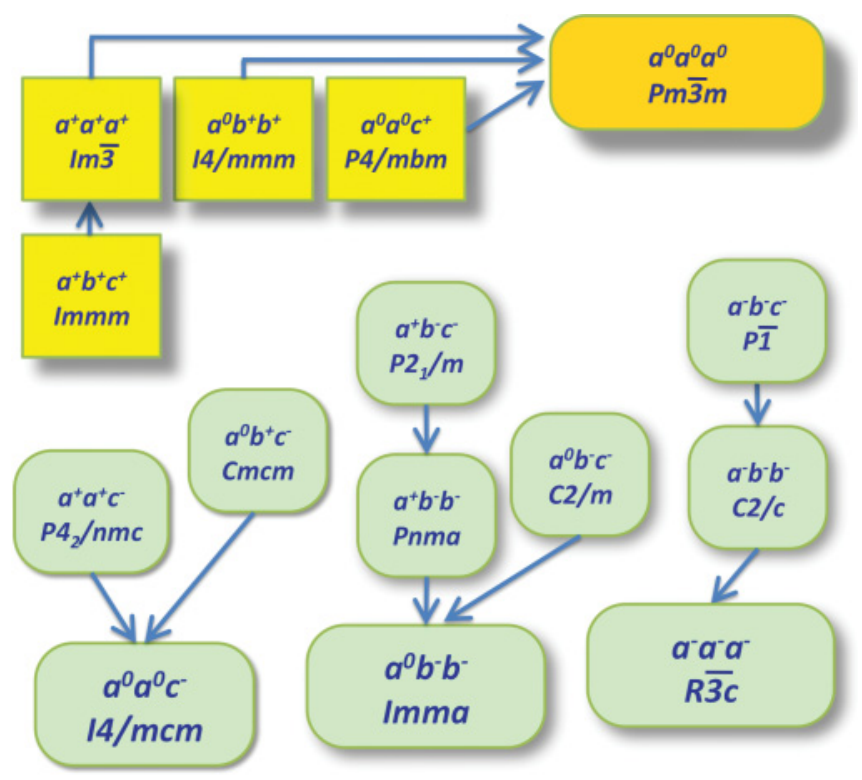

FIG. 3. (Color online) A schematic diagram, indicating the high-symmetry cubic perovskite structure and the possible 14 subgroup structures, which are the superpositions of oxygen octahedra rotations. ${ }^{42}$ Superpositions are indicated by Glazer's notations together with the corresponding crystalline symmetries. The structures indicated by rectangles relax to ideal cubic perovskite. The structures indicated by the rounded rectangles represent the families of tilted structures with energies lower than that of the ideal perovskite structure. Arrows indicate that a structure with lower symmetry relaxed to a higher-symmetry one. For example $P 2_{1} / m$ and its high-symmetry neighbor Pnma structure are both unstable and relax to Imma. Likewise the unstable $P \overline{1}$ and $C 2 / c$ structures relax to the $R \overline{3} c$ phase. 
summarized in Fig. 3. The following space group symmetries are allowed: $P m \overline{3} m, I m \overline{3}, I 4 / m m m, P 4 / m b m, I 4 / m c m$, Imma, $R \overline{3} c, I m m m, P 4_{2} / n m c, C m c m, P n m a, C 2 / m, C 2 / c$, $P 2_{1} / m$, and $P \overline{1}$. We calculated the total energies of each of these combinations, fully relaxing the ionic positions within the constraints of the chosen symmetry and with the unit cell
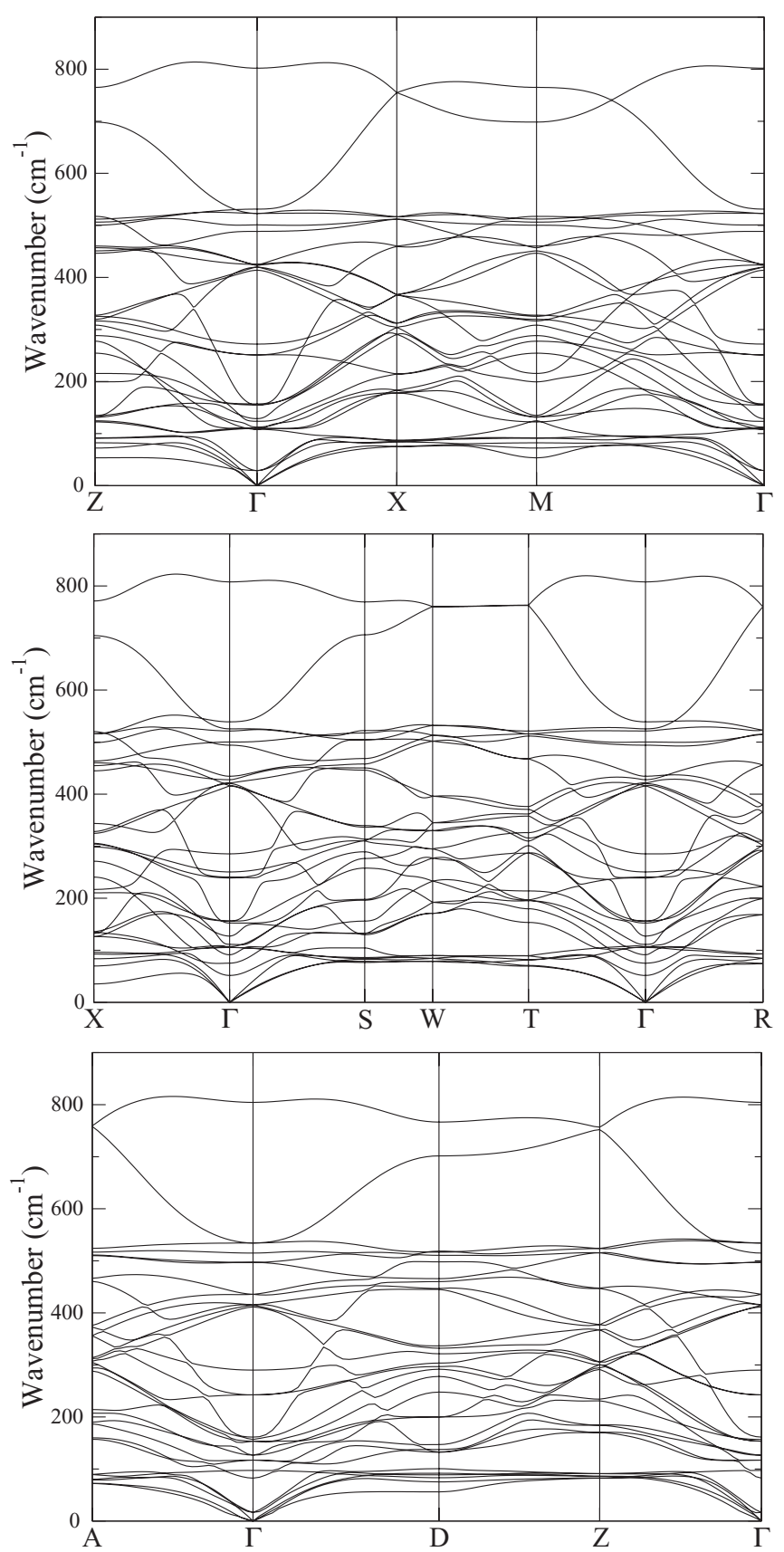

FIG. 4. Full phonon spectra of $\mathrm{EuTiO}_{3}$ in $I 4 / \mathrm{mcm}$ (upper panel), Imma (middle panel), and $R \overline{3} c$ (lower panel) structures. G-type AFM ordering of Eu magnetic moments was used in each case. All three structures are dynamically stable. In units of the reciprocal lattice vectors, the high-symmetry points are as follows. $14 / \mathrm{mcm}$ : $\mathrm{X}=$ $\left(0, \frac{1}{2}, 0\right), \mathrm{M}=\left(\frac{1}{2}, \frac{1}{2}, 0\right), \mathrm{Z}=\left(\frac{1}{2}, \frac{1}{2}, \frac{1}{2}\right)$. Imma: $\mathrm{X}=\left(\frac{1}{2}, \frac{1}{2},-\frac{1}{2}\right), \mathrm{S}=$ $\left(\frac{1}{2}, 0,0\right), \mathrm{R}=\left(0, \frac{1}{2}, 0\right), \mathrm{T}=\left(0,0, \frac{1}{2}\right), \mathrm{W}=\left(\frac{1}{4}, \frac{1}{4}, \frac{1}{4}\right) . R \overline{3} c: \mathrm{A}=\left(\frac{1}{2}, 0,0\right)$, $\mathrm{D}=\left(\frac{1}{2}, \frac{1}{2}, 0\right), \mathrm{Z}=\left(\frac{1}{2}, \frac{1}{2}, \frac{1}{2}\right)$. volume set to the experimental value. The shape of the unit cell was allowed to change according to the symmetry constraints.

We found that many of the low-symmetry tilt patterns relaxed to higher symmetry structures, as indicated by the arrows in Fig. 3. Three structures remained stable: those with $R \overline{3} c$, Imma, and $I 4 / \mathrm{mcm}$ space groups. All three are stabilized by around $25 \mathrm{meV}$ per formula unit compared with the prototype $P m \overline{3} m$ structure. The $I 4 / \mathrm{mcm}$ is lowest in energy ( $\sim 27 \mathrm{meV}$ per formula unit lower than $P m \overline{3} \mathrm{~m}$ ), Imma is the second most stable structure $(\sim 26 \mathrm{meV}$ per formula unit lower), then $R \overline{3} c$ at $\sim 25 \mathrm{meV}$ per formula unit lower. G-type antiferromagnetic configuration was found to be of lower energy than the ferromagnetic one in all tilted phases. Importantly, the energy differences between the $I 4 / \mathrm{mcm}$, Imma, and $R \overline{3} c$ structures are very small, within $\sim 2 \mathrm{meV}$ per formula unit, which is the range of numerical errors. Therefore we can not predict which rotation scenario is the ground state of $\mathrm{EuTiO}_{3}$. All three structures have no imaginary phonons through the entire Brillouin zone (see Fig. 4) and so are dynamically stable. Note that the $I 4 / \mathrm{mcm}$ structure is obtained by condensing out-of-phase rotations of oxygen octahedra about only one axis (see Fig. 5), Imma results from the same out-of-phase rotations about two axes, and $R \overline{3} c$ from out-of-phase rotations about all three axes. Our calculated structural parameters for all phases are collected in Table III.

To check whether our conclusions on the phase stability are affected by volume, we next investigated the athermal equation of state for the three candidate ground-state phases, as well as for the undistorted one. We found that the $E(V)$ dependence is adequately described by means of the first-order Murnaghan equation of state: ${ }^{44}$

$$
E=E_{0}+\frac{B_{0} V}{B_{0}^{\prime}}\left[\frac{\left(V_{0} / V\right)^{B_{0}^{\prime}}}{B_{0}^{\prime}-1}+1\right]-\frac{B_{0} V_{0}}{B_{0}^{\prime}-1},
$$

where $B_{0}$ is the bulk modulus, $B_{0}^{\prime}$ its pressure derivative, $V_{0}$ the equilibrium volume, and $E_{0}$ is the energy minimum corresponding to the equilibrium volume. Our calculated parameters for this equation, obtained by fitting of $a b$ initio

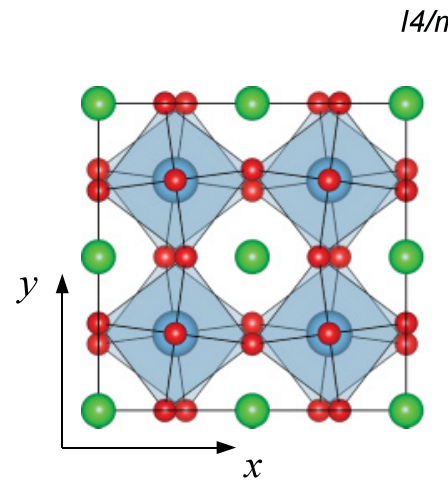

$4 / \mathrm{mcm}$

FIG. 5. (Color online) Schematic representation of $\mathrm{EuTiO}_{3}$ in $I 4 / \mathrm{mcm}$ space group structure with oxygen octahedra tilting. Left: view along the $z$ direction, where the out-of-phase tilting occurs, right: view along the $y$ direction without any tilting. The amount of tilting corresponds to the one obtained in calculations. Drawings were produced by VESTA visualization software. ${ }^{43}$ 
TABLE III. Theoretical structural parameters of $\mathrm{EuTiO}_{3}$ for $a^{0} a^{0} c^{-}(I 4 / m c m), a^{0} b^{-} b^{-}($Imma $)$and $a^{-} a^{-} a^{-}(R \overline{3} c)$ oxygen octahedral rotation patterns.

\begin{tabular}{|c|c|c|c|c|}
\hline & Wyckoff & $x$ & $y$ & $z$ \\
\hline \multicolumn{5}{|c|}{$\mathrm{I} 4 / \mathrm{mcm}$} \\
\hline $\mathrm{Eu}$ & $2(b)$ & 0 & 0.5 & 0.25 \\
\hline $\mathrm{Ti}$ & $2(c)$ & 0 & 0 & 0 \\
\hline $\mathrm{O}(1)$ & $2(a)$ & 0 & 0 & 0.25 \\
\hline $\mathrm{O}(2)$ & $4(h)$ & 0.21578 & 0.71578 & 0 \\
\hline \multicolumn{5}{|c|}{ Cell parameters } \\
\hline & $a(\AA)$ & 5.494 & & \\
\hline & $c(\AA)$ & 7.861 & & \\
\hline \multicolumn{5}{|c|}{ Imma } \\
\hline $\mathrm{Eu}$ & $4(e)$ & 0 & 0.25 & 0.00221 \\
\hline $\mathrm{Ti}$ & $4(b)$ & 0 & 0 & 0.5 \\
\hline $\mathrm{O}(1)$ & $4(e)$ & 0 & 0.25 & 0.54663 \\
\hline $\mathrm{O}(2)$ & $8(g)$ & 0.25 & 0.02415 & 0.25 \\
\hline \multicolumn{5}{|c|}{ Cell parameters } \\
\hline & $a(\AA)$ & 5.509 & & \\
\hline & $b(\AA)$ & 7.774 & & \\
\hline & $c(\AA)$ & 5.541 & & \\
\hline \multicolumn{5}{|c|}{$R \overline{3} c$} \\
\hline $\mathrm{Eu}$ & $2(a)$ & 0 & 0 & 0.25 \\
\hline $\mathrm{Ti}$ & $2(b)$ & 0 & 0 & 0 \\
\hline $\mathrm{O}$ & $6(e)$ & 0.53813 & 0 & 0.25 \\
\hline \multicolumn{5}{|c|}{ Cell parameters } \\
\hline & $a(\AA)$ & 5.528 & & \\
\hline & $c(\AA)$ & 13.448 & & \\
\hline
\end{tabular}

total energies, are listed in Table IV. The equilibrium values for $V_{0}, E_{0}$ and bulk modulus $B_{0}$ for tetragonal, orthorhombic and rhombohedral phases are almost the same, however, the pressure derivative $B_{0}^{\prime}$ for $R \overline{3} c$ phase is about 1.5 times lower then for the $I 4 / \mathrm{mcm}$ phase. This indicates that the internal relaxations in the rhombohedral phase are less sensitive to the volume changes than in the tetragonal phase. Also, the $R \overline{3} c$ structure is more rigid than the cubic and the tetragonal ones. Measurements of the bulk moduli and their pressure derivatives at different temperatures would be helpful in distinguishing between these lower-symmetry phases.

\section{IR optical properties in structures with tilting}

Finally, we attempt to distinguish between the three candidate ground-state structures by comparing their calculated phonon and mode-plasma frequencies with the measured

TABLE IV. Calculated Murnaghan equation of state parameters for the $I 4 / \mathrm{mcm}$, Imma, and $R \overline{3} \mathrm{c}$ structures. $V_{0}$ is the theoretical volume and $a_{0}=\left(V_{0}\right)^{1 / 3}$ is the theoretical pseudocubic lattice constant.

\begin{tabular}{lcccc}
\hline \hline & $P m \overline{3} m$ & $I 4 / m c m$ & Imma & $R \overline{3} c$ \\
\hline$V_{0}\left(\AA^{3}\right)$ & 61.29 & 61.04 & 61.11 & 61.08 \\
$a_{0}(\AA)$ & 3.943 & 3.937 & 3.939 & 3.938 \\
$B_{0}(\mathrm{GPa})$ & 162.5 & 171.1 & 169.4 & 179.1 \\
$B_{0}^{\prime}$ & 10.82 & 7.57 & 4.98 & 4.55 \\
\hline \hline
\end{tabular}

optical properties. We find that the calculated wave numbers of the $R \overline{3} c$ structure show best agreement with experiment, suggesting it as a likely experimental structure.

Our results are summarized in Tables IIa and IIb as well as in Fig. 6. In all cases, we used the Born effective charges calculated for the $P m \overline{3} m$ reference structure in our evaluation of the mode plasma frequencies, and our reported values do not include the LO-TO splitting. Calculations were performed for the G-type AFM ordered phase; the coupling between phonon properties and magnetism is discussed at the end of this section.

First, we look at the wave number of the soft TO1 mode (see Table IIa). Our calculated values are 107 and $128 \mathrm{~cm}^{-1}$ for $I 4 / \mathrm{mcm}$ (the symmetry lowering splits the TO1 mode into one doubly degenerate and one singly degenerate $c$ polarized mode), 80 and $124 \mathrm{~cm}^{-1}(R \overline{3} c)$, and 98 to $131 \mathrm{~cm}^{-1}$ (Imma). The experimental value is $82 \mathrm{~cm}^{-1}$, suggesting that the $R \overline{3} c$ structure is most likely. We note, however, that the soft-mode wave number is very sensitive to the volume of the structure and small changes in choice of volume (GGA versus experimental for example) can strongly change the predicted values. Regarding the mode-plasma frequencies of this mode, the experimental value is $1550 \mathrm{~cm}^{-1}$, and the average calculated values for the three structures are $1417 \mathrm{~cm}^{-1}$ $(I 4 / \mathrm{mcm}), 1433 \mathrm{~cm}^{-1}(R \overline{3} \mathrm{c})$, and $1382 \mathrm{~cm}^{-1}($ Imma $)$ all showing comparable agreement with the experimental value. Note that the relatively large mode splittings that we obtain for TO1 modes in the lower symmetry structures could explain the experimentally observed large peak width in the imaginary component of the complex dielectric function [see Fig. 3(b) in Ref. 24] when two modes with lifted degeneracy are fitted as one mode.

For completeness, we also list in Tables IIa and IIb our calculated frequencies and mode plasma frequencies of the TO2- and TO4-like modes. There are no striking differences that provide persuasive evidence for one structure over another. In the $I 4 / \mathrm{mcm}$ structure, the splitting of the TO2 mode, which is caused by the symmetry lowering, is small (see modes 3 and 4 in Table IIa). However, the mode-plasma frequencies of the symmetry-split TO2 modes are significantly different: mode 3 consists mainly of out-of-phase shifts of $\mathrm{Eu}$ and $\mathrm{Ti}$ atoms, without oxygen contribution, whereas the $c$-axis-polarized mode four has nonzero oxygen displacements that reduce the relative shifts of the $\mathrm{Ti}$ atoms, resulting in a lower dipole for this mode. The average value of the mode-plasma frequencies for these three modes is $703 \mathrm{~cm}^{-1}$, which is significantly lower than the corresponding value in the $P m \overline{3} m$ structure $\left(925 \mathrm{~cm}^{-1}\right)$ but still much higher than experimental one $\left(400 \mathrm{~cm}^{-1}\right)$. The average value of the mode-plasma frequency for the TO4 mode (modes 8 and 9) is $727 \mathrm{~cm}^{-1}$, compared with $820 \mathrm{~cm}^{-1}$ for the reference $P m \overline{3} m$ structure. In the $R \overline{3} c$ phase, the TO2 mode also splits by a small amount $\left(7 \mathrm{~cm}^{-1}\right)$ with average mode-plasma-frequency of $704 \mathrm{~cm}^{-1}$, and the TO4 mode averaged mode-plasma frequency is $734 \mathrm{~cm}^{-1}$; both values are similar to those of the $I 4 / \mathrm{mcm}$ structure. Like in the $I 4 / \mathrm{mcm}$ structure, mode 1 is built by Eu displacements against oxygen cage, with small in-phase contribution of $\mathrm{Ti}$ sublattice. The eigenvectors are also similar to those of the $P m \overline{3} m$ and $I 4 / m c m$ structures. In the Imma structure (see Table IIb), the wave numbers of the TO2-group are in the 


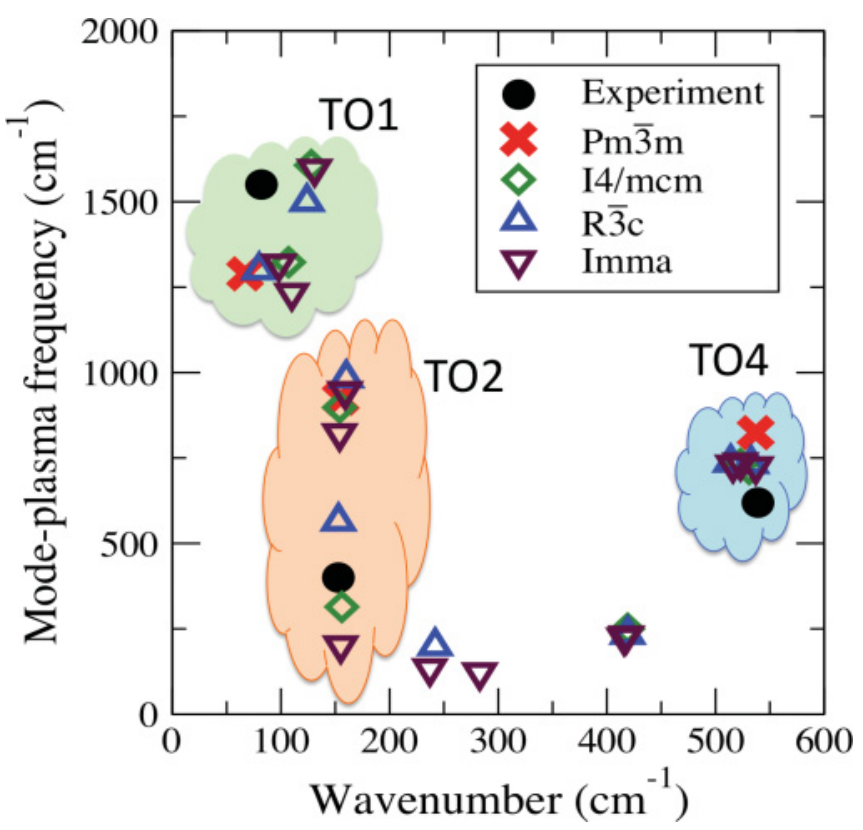

FIG. 6. (Color online) Calculated and measured ${ }^{24}$ mode-plasma frequencies, plotted as a function of the mode wave numbers. Our calculated values for four different structural symmetries are presented. Values originating from the TO1, TO2, and TO4 modes of the ideal perovskite are grouped together; the ungrouped symbols correspond to additional modes that appear due to the symmetry lowering.

range $154-159 \mathrm{~cm}^{-1}$, and those of the TO4 group are in the range $516-537 \mathrm{~cm}^{-1}$. As in the $R \overline{3} c$ structure, modes in the range $237-283 \mathrm{~cm}^{-1}$ are activated and should be observed as a doublet with a splitting of $50 \mathrm{~cm}^{-1}$.

One interesting observation is the presence of an IR-active mode with wave number $\sim 419 \mathrm{~cm}^{-1}$ in all three structures (mode 7 in Table IIa and 18 and 19 in Table IIb). This two-fold degenerate mode (it is a doublet in the low-symmetry Imma structure) has a relatively low mode-plasma frequency of $250 \mathrm{~cm}^{-1}$ (and, correspondingly, a low oscillator strength). The eigenvector of this mode shows that this vibration originates from the out-of-phase displacements of $\mathrm{Ti}$ atoms in the neighboring sublattices. However, the atomic displacements are not collinear, which leads to an uncompensated dipole in the plane, orthogonal to the $c$-axis. A mode with a similar wave number $\left(\sim 430 \mathrm{~cm}^{-1}\right)$ is observed at low temperatures in the experimental IR reflectivity data (see Fig. 2 in Ref. 24). The fact that experimentally this mode is observable only at low temperatures gives an additional indication that it could be a fingerprint of the antiferrodistortive phase transition.

To make the comparison with the available experimental data easier, we show in Fig. 6 the calculated and experimental mode-plasma frequencies as a function of wave number. Clearly, the calculated values for the $P m \overline{3} m$ structure are strikingly different from the experimental values providing further evidence that this is unlikely to be the ground-state structure. It remains difficult to distinguish between the $I 4 / \mathrm{mcm}, R \overline{3} \mathrm{c}$, and Imma structures that all show similarly good agreement with current experimental data. Our predicted differences in the degeneracies and ordering of the components of the TO1 and TO2 modes, could, in principle, be distinguished experimentally using polarized optical spectroscopy on single crystals.

\section{Magnetostructural coupling}

Strong magnetostructural coupling and multiferroic effects were predicted previously for $P m \overline{3} m \mathrm{EuTiO}_{3} ;{ }^{16}$ here we investigate whether such behavior persists in our newly predicted tilted ground-state structures. Indeed, we find that constraining the spins to be aligned ferromagnetically decreases the softmode wave number by $\sim 7 \mathrm{~cm}^{-1}$ for all three tilted structures; this is the same as previously calculated for the $P m \overline{3} m$ structure ${ }^{16}\left(7 \mathrm{~cm}^{-1}\right)$, and close to the value estimated from the low-temperature IR reflectivity spectra at different magnetic fields $\left(3 \mathrm{~cm}^{-1}\right) \cdot{ }^{23}$ We obtain a decrease of about $2 \mathrm{~cm}^{-1}$ for all modes belonging to the TO2 group. The wave number of the TO4 Axe mode is almost insensitive to the magnetic ordering. The modes corresponding to tilting of the oxygen octahedra, on the other hand, show strong magneto-phonon coupling, since they cause changes in the Eu-O-Eu angles which in turn strongly modify the superexchange pathways between the magnetic ions. These modes are listed in Table IIa as mode 5. We find that ferromagnetic constraint on the Eu spins hardens this mode in the $I 4 / \mathrm{mcm}$ structure from 164 to $218 \mathrm{~cm}^{-1}$, whereas in the $R \overline{3} c$ structure it is softened by $17 \mathrm{~cm}^{-1}$. Since the tilt mode is not polar, however, the dielectric function is not affected.

Finally, we mention that Shvartsman et al. ${ }^{10}$ recently measured an unusual off-diagonal magnetoelectric coupling in $\mathrm{EuTiO}_{3}$, which they rationalized using our predictions of tilted ground states. In their experiments, the applied electric field breaks inversion symmetry, allowing a DzyaloshinskiiMoriya interaction and subsequent magnetoelectric response analogous to that in perovskite $\mathrm{FeTiO}_{3}$, which also shows magnetic A-site ions and octahedral tiltings. ${ }^{45,46}$

\section{CONCLUSIONS}

In summary, our density functional calculations for perovskite $\mathrm{EuTiO}_{3}$ predict that its ground state consists of tilts and rotations of the oxygen octahedra, rather than the simple cubic perovskite structure as previously believed. By comparing the total energies of all symmetry allowed tilting patterns, we identified three candidate ground states: $a^{0} a^{0} c^{-}(I 4 / \mathrm{mcm})$, $a^{0} b^{-} b^{-}$(Imma), and $a^{-} a^{-} a^{-}(R \overline{3} c)$. We compared the calculated phonon properties with available experimental data for the three candidate structures to identify the most likely ground state. Our calculated energy differences are too small to allow us to predict which of these phases is the ground state; indeed, a phase coexistence might be possible, and additional experiments are required for further progress. In particular, a search for the infra-red-active mode in the range $220-290 \mathrm{~cm}^{-1}$ is a signature of $R \overline{3} c$ and Imma structures, but is not visible in $I 4 / \mathrm{mcm}$ phase. Also the magnetic-field dependence of the mode at $\sim 164 \mathrm{~cm}^{-1}$ would distinguish between the $I 4 / \mathrm{mcm}$ and $R \overline{3} c$ structures.

\section{ACKNOWLEDGMENTS}

This work was supported by the Young Investigators Group Programme of the Helmholtz Association, Germany, contract 
VH-NG-409 and by the ETH Zürich. We thank S. Kamba, R. Hermann, J. Hlinka, W. Kleemann, C. Fennie, S. Blügel, and
I. Slipukhina for discussions and gratefully acknowledge the support of Jülich Supercomputing Centre.
${ }^{1}$ T. R. McGuire, M. W. Shafer, R. J. Joenk, H. A. Alperin, and S. J. Pickart, J. Appl. Phys. 37, 981 (1966).

${ }^{2}$ G. McCarthy, W. White, and R. Roy, J. Inorg. Nucl. Chem. 31, 329 (1969).

${ }^{3}$ C.-L. Chien, S. DeBenedetti, and F. D. S. Barros, Phys. Rev. B 10, 3913 (1974).

${ }^{4}$ S. Chae, Y. Chang, D.-W. Kim, B. Lee, I. Choi, and C. Jung, J. Electroceram. 22, 216 (2009).

${ }^{5}$ T. Katsufuji and H. Takagi, Phys. Rev. B 64, 054415 (2001).

${ }^{6}$ S. J. Gong and Q. Jiang, Phys. Status Solidi B 241, 3033 (2004).

${ }^{7}$ Q. Jiang and H. Wu, J. Appl. Phys. 93, 2121 (2003).

${ }^{8}$ H. Wu and W. Shen, Solid State Commun. 133, 487 (2005).

${ }^{9}$ H. Wu, Q. Jiang, and W. Shen, Phys. Lett. A 330, 358 (2004).

${ }^{10}$ V. V. Shvartsman, P. Borisov, W. Kleemann, S. Kamba, and

T. Katsufuji, Phys. Rev. B 81, 064426 (2010).

${ }^{11}$ G. J. Conduit and B. D. Simons, Phys. Rev. B 81, 024102 (2010).

${ }^{12}$ K. Fujita, N. Wakasugi, S. Murai, Y. Zong, and K. Tanaka, Appl. Phys. Lett. 94, 062512 (2009).

${ }^{13}$ H.-H. Wang, A. Fleet, J. D. Brock, D. Dale, and Y. Suzuki, J. Appl. Phys. 96, 5324 (2004).

${ }^{14}$ J. H. Lee, X. Ke, N. J. Podraza, L. F. Kourkoutis, T. Heeg, M. Roeckerath, J. W. Freeland, C. J. Fennie, J. Schubert, D. A. Muller, P. Schiffer, and D. G. Schlom, Appl. Phys. Lett. 94, 212509 (2009).

${ }^{15}$ K. Kugimiya, K. Fujita, K. Tanaka, and K. Hirao, J. Magn. Magn. Mater. 310, 2268 (2007).

${ }^{16}$ C. J. Fennie and K. M. Rabe, Phys. Rev. Lett. 97, 267602 (2006).

${ }^{17}$ J. Lee, L. Fang, E. Vlahos, X. Ke, Y. Jung, L. Kourkoutis, J. Kim, P. Ryan, T. Heeg, M. Roeckerath, V. Goian, M. Bernhagen, R. Uecker, P. Hammel, K. Rabe, S. Kamba, J. Schubert, J. Freeland, D. Muller, C. Fennie, P. Schiffer, V. Gopalan, E. Johnston-Halperin, and D. Schlom, Nature (London) 466, 954 (2010).

${ }^{18}$ H. Wu, Q. Jiang, and W. Z. Shen, Phys. Rev. B 69, 014104 (2004).

${ }^{19}$ K. Z. Rushchanskii, S. Kamba, V. Goian, P. Vaňek, M. Savinov, J. Prokleška, D. Nuzhnyy, K. Knížek, F. Laufek, S. Eckel, S. K. Lamoreaux, A. O. Sushkov, M. Ležaić, and N. A. Spaldin, Nat. Mater. 9, 649 (2010).

${ }^{20}$ R. Ranjan, H. S. Nabi, and R. Pentcheva, J. Phys. Condens. Matter 19, 406217 (2007).

${ }^{21}$ R. Ranjan, H. S. Nabi, and R. Pentcheva, J. Appl. Phys. 105, 053905 (2009).

${ }^{22}$ A. Bussmann-Holder, J. Köhler, R. K. Kremer, and J. M. Law, Phys. Rev. B 83, 212102 (2011).
${ }^{23}$ S. Kamba, D. Nuzhnyy, P. Vaněk, M. Savinov, K. Knížek, Z. Shen, E. Šantavá, K. Maca, M. Sadowski, and J. Petzelt, Europhys. Lett. 80, 27002 (2007).

${ }^{24}$ V. Goian, S. Kamba, J. Hlinka, P. Vaněk, A. A. Belik, T. Kolodiazhnyi, and J. Petzelt, Eur. Phys. J. B 71, 429 (2009).

${ }^{25}$ J. Hlinka, J. Petzelt, S. Kamba, D. Noujni, and T. Ostapchuk, Phase Transitions 79, 41 (2006).

${ }^{26}$ R. I. Eglitis, A. V. Postnikov, and G. Borstel, Phys. Rev. B 54, 2421 (1996).

${ }^{27}$ J. P. Perdew, K. Burke, and M. Ernzerhof, Phys. Rev. Lett. 77, 3865 (1996).

${ }^{28}$ G. Kresse and J. Hafner, Phys. Rev. B 47, 558 (1993).

${ }^{29}$ G. Kresse and J. Furthmüller, Phys. Rev. B 54, 11169 (1996).

${ }^{30}$ P. E. Blöchl, Phys. Rev. B 50, 17953 (1994).

${ }^{31}$ G. Kresse and D. Joubert, Phys. Rev. B 59, 1758 (1999).

${ }^{32}$ V. I. Anisimov, F. Aryasetiawan, and A. I. Lichtenstein, J. Phys. Condens. Matter 9, 767 (1997).

${ }^{33}$ S. L. Dudarev, G. A. Botton, S. Y. Savrasov, C. J. Humphreys, and A. P. Sutton, Phys. Rev. B 57, 1505 (1998).

${ }^{34}$ K. Kunc and R. M. Martin, Phys. Rev. Lett. 48, 406 (1982).

${ }^{35}$ D. Alfè, Comput. Phys. Commun. 180, 2622 (2009).

${ }^{36}$ P. Ghosez, J.-P. Michenaud, and X. Gonze, Phys. Rev. B 58, 6224 (1998).

${ }^{37}$ P. Ghosez, E. Cockayne, U. V. Waghmare, and K. M. Rabe, Phys. Rev. B 60, 836 (1999).

${ }^{38}$ R. D. King-Smith and D. Vanderbilt, Phys. Rev. B 47, 1651 (1993).

${ }^{39}$ Note that one suggestion explaining the origin of the mixing of the $\mathrm{TO} 1$ and TO2 modes is a superexchange mechanism between $\mathrm{Eu}^{2+}$ 4f spins via the $3 d$ states of nonmagnetic $\mathrm{Ti}^{4+}$, see $\mathrm{H}$. Akamatsu, Y. Kumagai, F. Oba, K. Fujita, H. Murakami, K. Tanaka, and I. Tanaka, Phys. Rev. B 83, 214421 (2011).

${ }^{40}$ C. Lasota, C.-Z. Wang, R. Yu, and H. Krakauer, Ferroelectrics 194, 109 (1997).

${ }^{41}$ A. M. Glazer, Acta Crystallogr. Sect. B 28, 3384 (1972).

${ }^{42}$ C. J. Howard and H. T. Stokes, Acta Crystallogr. Sect. B 54, 782 (1998).

${ }^{43}$ K. Momma and F. Izumi, J. Appl. Crystallogr. 44, 1272 (2011).

${ }^{44}$ F. D. Murnaghan, PNAS 30, 244 (1944).

${ }^{45}$ C. J. Fennie, Phys. Rev. Lett. 100, 167203 (2008).

${ }^{46}$ T. Varga, A. Kumar, E. Vlahos, S. Denev, M. Park, S. Hong, T. Sanehira, Y. Wang, C. J. Fennie, S. K. Streiffer, X. Ke, P. Schiffer, V. Gopalan, and J. F. Mitchell, Phys. Rev. Lett. 103, 047601 (2009). 